\title{
Cervical Intervertebral Disc Displacement in Multiple Sclerosis Patients: A Possible Trigger for the Pathogenesis of Multiple Sclerosis?
}

\author{
Andre Eduardo de Almeida Franzoia, c, Mariana Heil Kinas ${ }^{\mathrm{a}}$, Matheus Dorneles Fronza ${ }^{\mathrm{a}}$, \\ Francisco Virmond Moreira ${ }^{\mathrm{a}}$, Paulo Roberto Wille ${ }^{\mathrm{b}}$
}

\begin{abstract}
Background: Few articles in the world have already correlated these two themes. The reason we wrote this paper is to suggest a pathogenic hypothesis that correlates the two diseases.

Methods: We reviewed the medical literature in the MEDLINE/PubMed and LILACS/SciELO databases. We used the terms "cervical intervertebral disc displacement", "multiple sclerosis", "neuroinflammation", "pathogenesis" and its combinations.

Results: Multiple sclerosis (MS) is an inflammatory demyelinating disease of the central nervous system (CNS). Spinal cord myelopathy in MS presents with neck and radicular pain, segmental weakness, hypoesthesia and diminution of tendon reflexes. All of these symptoms may also appear in myelopathy due to cervical spine compression. It is observed in the context of cervical intervertebral disc displacement (CIDD). CIDD is the most common progressive disorder in the aging cervical spine. Clinical researches have described CIDD at different levels in MS patients. However, the majority of these clinical studies were only descriptive. The correlation between the two diseases and the study of the pathogenesis of them was little studied. We hypothesized an unprecedented theory for a possible pathogenic mechanism between CIDD and MS. Can the CIDD be a trigger for the onset of spinal demyelination in MS patients?
\end{abstract}

Conclusions: We believe with blood-spinal cord barrier (BSCB) disruption the neutrophils invasion on CNS is facilitated. Thus local neuroinflammation on the spinal cord in patients with CIDD could be a facilitating factor for neutrophils infiltration to the CNS in MS. This cascade of neuroinflammation may be one of the triggers for the activation of demyelination in the spinal cord of MS patients.

Manuscript submitted April 6, 2019, accepted May 24, 2019

${ }^{a}$ Department of Medicine, University of the Region of Joinville (UNIVILLE), Joinville SC, Brazil

${ }^{b}$ University of the Region of Joinville (UNIVILLE), Joinville SC, Brazil

'Corresponding Author: Andre Eduardo de Almeida Franzoi, Padre Kolb, 1273-Joinville, Santa Catarina 89202350, Brazil.

Email: andrefranzoi@hotmail.com

doi: https://doi.org/10.14740/jnr533
Keywords: Cervical intervertebral disc displacement; Multiple sclerosis; Neuroinflammation; Pathogenesis

\section{Introduction}

Multiple sclerosis (MS) is an inflammatory demyelinating disease of the central nervous system (CNS). The disease is usually diagnosed in young adults aged 30 to 40 years. Focal lymphocytic infiltration leads to myelin and axonal damage. Initially, inflammation is transient and remyelination occurs but is not durable. MS is characterized by multiple lesions of the brain and spinal cord. These lesions are disseminated in time and space. The onset of the disease is characterized by episodes of neurological dysfunction that usually recover. Over time, pathological changes become dominated by generalized microglial activation, associated with extensive, neuroinflammation and chronic demyelination. The patients accumulate progressive disability [1].

Spinal cord myelopathy in MS presents with neck and radicular pain, segmental weakness, hypoesthesia and diminution of tendon reflexes. All of these symptoms may also appear in myelopathy due to cervical spine compression. It is observed in the context of cervical intervertebral disc displacement (CIDD) [2]. CIDD is the most common progressive disorder in the aging cervical spine. It results from the process of degeneration of the intervertebral discs and facet joints of the cervical spine. Biomechanically, the disc and the facets are the connecting structures between the vertebrae for the transmission of external forces. They also facilitate cervical spine mobility [3].

The disease presents mainly in people aged over 50 years. Over $60 \%$ of people over 50 years have some degree of neurological abnormality related to myelopathy secondary to CIDD. According Manchikanti L et al, CIDD are the main etiology of pain in the spine of the secondary type. It mainly affects the male population between the fourth and fifth decade of life. Manchikanti L and colleagues described the annual incidence of CIDD with radiculopathy is 83 to every 100,000 patients 13 to 91 years of age [4]. Cohen SP et al estimated a prevalence of cervical pain of $15-50 \%$. The author affirms the main cause of neck pain is CIDD [5]. 
Table 1. Five Articles Selected in a Non-Systematic Way

\begin{tabular}{lll}
\hline Main author & Title & Journal \\
\hline Xydis VG et al [11] & The association between multiple sclerosis and spondylosis: When and why & European Journal of Radiology \\
Russi AE et al [12] & The meninges: new therapeutic targets for multiple sclerosis & Translation Research \\
Uchida Y et al [13] & $\begin{array}{l}\text { Involvement of claudin-11 in disruption of blood-brain, -spinal cord, and } \\
\text {-arachnoid barriers in multiple sclerosis }\end{array}$ & Molecular Neurobiology \\
Aube B et al [14] & $\begin{array}{l}\text { Neutrophils mediate blood-spinal cord barrier disruption in demyelinating } \\
\text { neuroinflammatory diseases }\end{array}$ & The Journal of Immunology \\
Spencer JI et al [15] & $\begin{array}{l}\text { Vascular pathology in multiple sclerosis: reframing pathogenesis around the Journal of Neurology, Neurosurgery, and } \\
\text { blood-brain barrier }\end{array}$ & Psychiatry \\
\hline
\end{tabular}

Symptoms related to myelopathy and radiculopathy are caused by the formation of osteophytes. It compromises the diameter of the spinal cord canal. The developmental process with the degenerative process may cause mechanical pressure on the spinal cord. This compression can affect one or multiple levels of cervical spinal cord. This pressure may produce direct neurological damage or ischemic changes. It leads to spinal cord disturbances and neuroinflammation $[3,6,7]$.

CIDD is a prevalent disease and has high functional impact in the general population. However, the correlation of this disease and MS was little studied. In 1957, Brain R and colleagues reported the association between MS and CIDD in 17 patients. The patients were aged 37 to 61 years. The diagnosis of CIDD was made with X-rays and myelography. The diagnosis of MS was based on clinical evaluation and cerebrospinal fluid (CSF) analysis. Magnetic resonance imaging (MRI) is currently the gold standard modality for the evaluation of brain and spinal cord lesions in MS patients, and is also important for the assessment of myelopathy [8].

Clinical researches have described CIDD at different levels in MS patients $[9,10]$. But these clinical studies were only descriptive. These researches did not evaluate pathological mechanisms to correlate CIDD with MS. The correlation between the two diseases was little studied.

\section{Material and Methods}

We reviewed the medical literature in the MEDLINE/PubMed and LILACS/SciELO databases. We used the terms "cervical intervertebral disc displacement", "multiple sclerosis", "neuroinflammation", "pathogenesis", and its combinations.

Since this was a literature review based study, the Institutional Review Board (IRB) approval and ethical compliance with human/animal study are not applicable.

\section{Results}

After the electronic search in the databases, we selected five articles in a non-systematic way to write the discussion (Table 1, [11-15]). Few articles in the world have already correlated these two themes. This was the reason we wrote this paper, to suggest a pathogenic hypothesis that correlates the two diseases.

\section{Discussion}

A pathogenic mechanism correlating CIDD and MS has been proposed only once in the medical literature. Xydis VG et al evaluated risk factors for the development of CIDD in patients with MS [11]. The researchers distributed 84 individuals in two groups. 42 consecutive patients aged 23 to 66 years with MS and 42 age and sex matched controls were evaluated retrospectively. Clinical disability was evaluated with the expanded disability status scale (EDSS) and spasticity with the Asworth score [11].

Xydis VG and colleagues assessed total brain lesion volume (BLV), total grey matter (GM) volume and deep GM volume. In the cervical spine, the indices of CIDD (disc dehydration, disc protrusion, abnormal posture and osteophytosis) and the spinal cord injury load (SCIL) were evaluated. The authors described the association of CIDD indices with the presence of MS. Xydis VG et al affirmed the clinical scales, the brain and spinal cord imaging measurements were assessed [11].

According Xydis VG et al, presence of MS was positively associated with abnormal posture $(\mathrm{P}=0.002)$, disc dehydration at $\mathrm{C} 6-\mathrm{C} 7(\mathrm{P}=0.049)$ and posterior disc protrusion at $\mathrm{C} 5-\mathrm{C} 6$ $(\mathrm{P}=0.033)$ and $\mathrm{C} 6-\mathrm{C} 7(\mathrm{P}=0.001)$. The author affirmed all patients had spasticity. Patients with abnormal posture were younger $(37.5 \pm 11.1$ years $)$ than those with normal $(45.4 \pm$ 8.6years) $(\mathrm{P}=0.024)$. The factors age $(\mathrm{P}=0.008)$, EDSS $(\mathrm{P}=$ $0.045)$ and $\mathrm{BLV}(\mathrm{P}=0.084)$ were significant independent predictors of abnormal posture. Xydis VG et al described younger age combined with worse EDSS and increased BLV predicted abnormal posture [11].

Secondly Xydis VG and colleagues found MS patients presented CIDD more frequently. It is associated with younger age, more severe disability and extensive lesions in the brain. For Xydis VG et al, spasticity induced by the brain lesions and abnormal expression of extracellular matrix proteins in the brain and the intervertebral disc constitute a possible pathogenic mechanism [11].

However, we believe the two diseases relate differently from the spasticity of MS patients. We agree there may be a correlation in the pathogenesis between CIDD and MS. Nevertheless, we hypothesized CIDD generates local inflammation through compression on the spinal cord. The stimulus of neuroinflammation on the spinal cord can be related to MS 
through the blood-spinal cord barrier (BSCB) breakdown.

Neuroinflammation factors on BSCB are being increasingly studied and discussed in MS $[12,13]$. Disruption of the BSCB and immune cell neuroinflammation are early pathogenic hallmarks of MS and animal model with experimental autoimmune encephalomyelitis (EAE) [14].

According Spencer JI et al, the BSCB may be more permeable than the blood-brain barrier (BBB). The spinal cord symptoms are commonly early in the disease course and become a predominant feature in the progressive phase [15].

Aube B et al induced EAE in lys-eGFP-ki mice and performed single and nonterminal intravital imaging to investigate BSCB permeability. It was investigated simultaneously with the kinetics of GFP (+) myeloid cell infiltration. The authors observed a loss in BSCB integrity within 1 day of disease onset. It occurred in parallel to the infiltration of GFP $(+)$ cells into the CNS and lasted for 4 days. Neutrophils accounted for a significant proportion of the circulating and CNS-infiltrating myeloid cells during the preclinical phase of EAE. The depletion of these cells delayed the onset and reduced the severity of EAE while maintaining BSCB integrity [14].

Aube B and colleagues showed neutrophils collected from the blood or bone marrow of EAE mice transmigrate more efficiently than from neutrophils of naive animals in a BBB cell culture model. Aube B et al used intravital videomicroscopy and demonstrated the interleukin (IL)-1R type 1 governing the adhesion of neutrophils to the inflamed spinal cord vasculature. The immunostaining of postmortem CNS material obtained from an acutely ill MS patient revealed instance of infiltrated neutrophils associated with regions of $\mathrm{BBB}$ or BSCB leakage. Their data provide evidence that neutrophils are involved in the initial events that take place during EAE. These cells are intimately linked with the status of the BBB and BSCB [14].

We believe with BSCB disruption the neutrophils invasion on CNS is facilitated. Thus local neuroinflammation on the spinal cord in patients with CIDD could be a facilitating factor for neutrophils infiltration to CNS in MS. This cascade of neuroinflammation may be one of the triggers for the activation of demyelination in the spinal cord of MS patients. The Figure 1 summarizes this hypothesis. We suggest and encourage further preclinical and clinical studies assess the BSCB breakdown hypothesis as a trigger for early demyelination in MS.

\section{Acknowledgments}

None to declare.

\section{Financial Disclosure}

None to declare.

\section{Conflict of Interest}

None to declare.
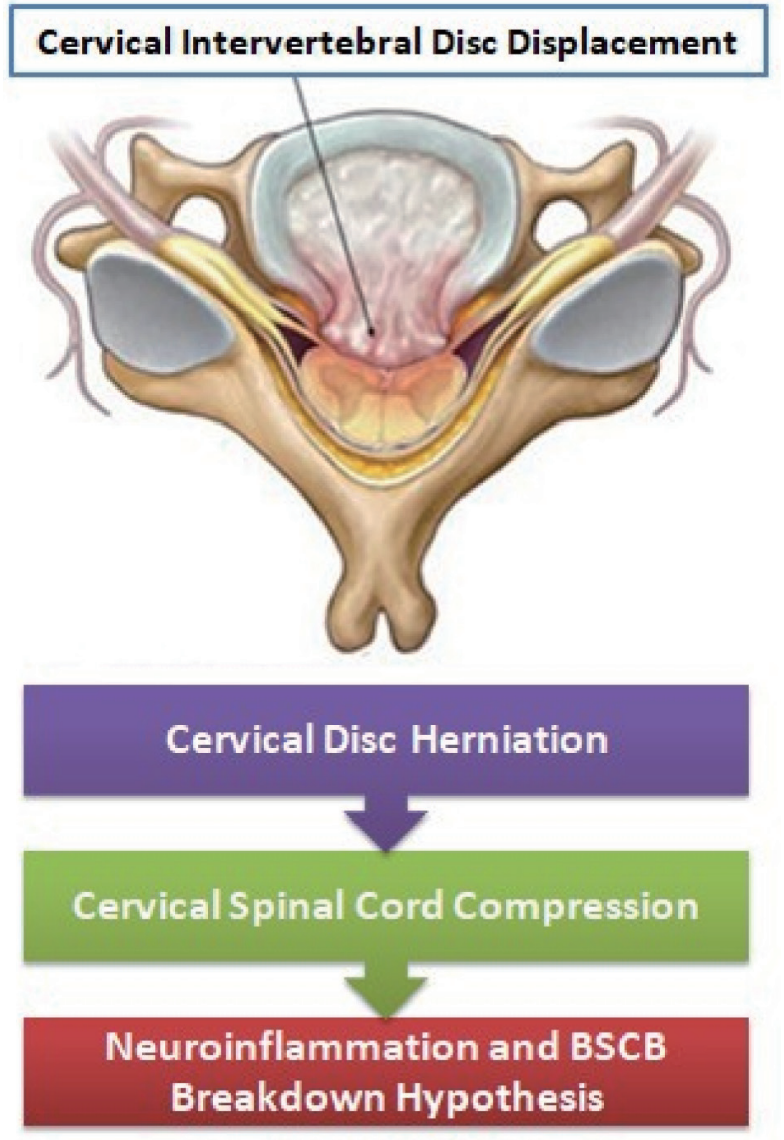

Figure 1. The BSCB breakdown hypothesis with neuroinflammation in MS patients after cervical spinal cord compression in CIDD. BSCB: blood-spinal cord barrier.

\section{Informed Consent}

Not applicable.

\section{Author Contributions}

Andre Eduardo de Almeida Franzoi designed the study, collected and analyzed the data, and wrote the article. Mariana Heil Kinas, Matheus Dorneles Fronza, Francisco Virmond Moreira and Paulo Roberto Wille analyzed the data and reviewed the article

\section{References}

1. Compston A, Coles A. Multiple sclerosis. Lancet. 2008;372(9648):1502-1517.

2. Brownlee WJ, Hardy TA, Fazekas F, Miller DH. Diagnosis of multiple sclerosis: progress and challenges. Lancet. 2017;389(10076):1336-1346.

3. Shedid D, Benzel EC. Cervical spondylosis anatomy: pathophysiology and biomechanics. Neurosurgery. 
2007;60(1 Supp1 1):S7-13.

4. Manchikanti L, Cash KA, Pampati V, Wargo BW, Malla Y. Management of chronic pain of cervical disc herniation and radiculitis with fluoroscopic cervical interlaminar epidural injections. Int J Med Sci. 2012;9(6):424-434.

5. Cohen SP. Epidemiology, diagnosis, and treatment of neck pain. Mayo Clin Proc. 2015;90(2):284-299.

6. Okada E, Matsumoto M, Ichihara D, Chiba K, Toyama Y, Fujiwara H, Momoshima S, et al. Aging of the cervical spine in healthy volunteers: a 10-year longitudinal magnetic resonance imaging study. Spine (Phila Pa 1976). 2009;34(7):706-712.

7. Matsumoto M, Okada E, Ichihara D, Watanabe K, Chiba $\mathrm{K}$, Toyama Y, Fujiwara H, et al. Age-related changes of thoracic and cervical intervertebral discs in asymptomatic subjects. Spine (Phila Pa 1976). 2010;35(14):1359-1364.

8. Brain R, Wilkinson M. The association of cervical spondylosis and disseminated sclerosis. Brain. 1957;80(4):456478.

9. Korovessis P, Maraziotis T, Stamatakis M, Baikousis A. Simultaneous three-level disc herniation in a patient with multiple sclerosis. Eur Spine J. 1996;5(4):278-280.

10. Young WF, Weaver M, Mishra B. Surgical outcome in pa- tients with coexisting multiple sclerosis and spondylosis. Acta Neurol Scand. 1999;100(2):84-87.

11. Xydis VG, Zikou AK, Kostadima V, Astrakas LG, Kosta $\mathrm{P}$, Argyropoulou MI. The association between multiple sclerosis and spondylosis: When and why. Eur J Radiol. 2017;91:47-51.

12. Russi AE, Brown MA. The meninges: new therapeutic targets for multiple sclerosis. Transl Res. 2015;165(2):255269.

13. Uchida Y, Sumiya T, Tachikawa M, Yamakawa T, Murata S, Yagi Y, Sato K, et al. Involvement of claudin-11 in disruption of blood-brain, -spinal cord, and -arachnoid barriers in multiple sclerosis. Mol Neurobiol. 2019;56(3):2039-2056.

14. Aube B, Levesque SA, Pare A, Chamma E, Kebir H, Gorina R, Lecuyer MA, et al. Neutrophils mediate bloodspinal cord barrier disruption in demyelinating neuroinflammatory diseases. J Immunol. 2014;193(5):24382454.

15. Spencer JI, Bell JS, DeLuca GC. Vascular pathology in multiple sclerosis: reframing pathogenesis around the blood-brain barrier. J Neurol Neurosurg Psychiatry. 2018;89(1):42-52. 